\title{
High-pressure structural stability of the ductile intermetallic compound, ErCu
}

\author{
S MEENAKSHI \\ High Pressure and Synchrotron Radiation Physics Division, Bhabha Atomic Research Centre, \\ Mumbai 400 085, India
}

MS received 10 July 2013; revised 16 August 2013

\begin{abstract}
High-pressure angle dispersive X-ray diffraction measurements up to 23.6 GPa have been carried out on the ductile intermetallic compound, $\mathrm{ErCu}$. Our measurements show that the ambient CsCl structure (SG: Pm-3m) is stable up to the highest pressure of the present measurements. A second-order BirchMurnaghan equation of state fit to the pressure, volume data yielded a bulk modulus of 67.6 GPa with the pressure derivative of bulk modulus fixed at 4 .
\end{abstract}

Keywords. Intermetallics; X-ray diffraction; high pressure; synchrotron radiation.

\section{Introduction}

Intermetallic compounds hold much potential to improve the performance of engineering systems owing to their low density, good oxidation resistance and high strength and stiffness at elevated temperature (Fleischer and Zabala 1990; Stoloff et al 2000). Commercial application of bulk intermetallic compounds is, however, limited largely because of their low ductility and low fracture toughness at room temperatures, which severely limits their fabricaion and their reliability in service.

Recently, however, high ductility at room temperature has been reported in fully ordered and stoichiometric B2-type (CsCl-type structure, SG: $\mathrm{Pm}$-3m) intermetallic compounds with RM composition (where $\mathrm{R}$ is a rareearth element, e.g. Y, Dy, Ho, Er, Ce and Nd; and $\mathrm{M}$ is a metal like $\mathrm{Cu}$ and Ag) (Gschneidner et al 2003). The crystal structure of these compounds comprises the corners of the unit cell cube occupied by the ' $M$ ' element and cube centre occupied by the ' $R$ ' element. Although some ductility has been reported in a small number of other B2 intermetallics, these rare earth B2 intermetallics are novel in that they are line compounds that tolerate no appreciable deviation from equimolar composition. Other intermetallics have been shown to be ductile when the composition is off stoichiometry (Takasugi et al 1987). Hence, these compounds hold promise of practical applications as they exhibit remarkable tensile elongation of the order of 20\% (Gschneidner et al 2003; Gschneidner 2009). The fracture toughness value for the RM compounds has been found to be very large (almost equivalent to that of aluminium alloys) and this has been

(smeena2001@gmail.com) attributed to their multiple slip systems, fine grain size and to the possible ' $R$ ' element counteracting environmental embrittlement (Zhang et al 2005). A large number of experimental and theoretical investigations have been carried out to examine various properties of these intermetallic compounds (Degtyareva et al 1997; Gschneidner et al 2003; Morris et al 2004; Demchyna et al 2006; Shi et al 2007; Wu et al 2008; Chouhan et al 2011; Tao et al 2011; Jun et al 2012; Wang et al 2012; Wu et al 2012). Theoretical calculations of the elastic constants of these RM intermetallics have shown that these materials possess $\mathrm{B} / \mathrm{G}$ values much larger than $1 \cdot 75$, the so-called ductility index proposed for a material to be ductile (Pugh 1954; Chouhan et al 2011; Wu et al 2012). On the experimental front, thermal expansion measurements on $\mathrm{HoCu}$ and DyCu have indicated anomaly near 26 and $61 \mathrm{~K}$, respectively, and these have been ascribed to the onset of antiferromagnetic order (Ibarra et al 1989; Amara et al 1997). The magnetic behaviour of these equiatomic RM ( $\mathrm{R}$ = rare earth, $\mathrm{M}=$ non-magnetic) compounds marked by a different type of magnetic ordering has been related to their conduction electron density. Thus, the changes in the electron density could lead to structural transformation in these compounds. In fact, a transformation from the ambient $\mathrm{CsCl}$ to orthorhombic $\mathrm{FeB}$ structure has been reported over a broad temperature range near the Neel temperature in a related compound $\mathrm{GdCu}$ using neutron diffraction (Blanco et al 1999). This phase transition marked by a large thermal hysteresis with pronounced anomalies in unit cell volume and electrical resistivity has been characterized as a diffusionless thermoelastic martensitic transformation. Recent results on $\mathrm{YCu}$ and $\mathrm{GdCu}$ obtained from X-ray absorption spectroscopy indicates that an $s$ to $d$ electron transfer and 
$d$-band broadening due to volume reduction plays a major role in driving the cubic-orthorhombic transition (Chaboy and Ibarra 1995).

In view of these interesting observations, detailed structural investigations on these materials could provide insights into understanding the material and its potential applications. It is well known that high pressure is a perfect tool to change the electronic and structural properties of materials in a controlled manner. In the present work, we have carried out high-pressure angle-dispersive X-ray diffraction (ADXRD) measurements on the ductile intermetallic compound ErCu up to 23.6 GPa with an aim to investigate its structural stability under compression.

\section{Experimental}

The compound ErCu was prepared by arc melting the constituent elements under argon atmosphere. The buttons were homogenized by repeated re-melting. Samples sealed in quartz tube under vacuum were further annealed at $700{ }^{\circ} \mathrm{C}$ for $24 \mathrm{~h}$. The structural characterization carried out using XRD indicated a cubic symmetry (SG: Pm-3m) with a lattice parameter of $3.430 \AA$. A very weak peak at $\sim 13.5^{\circ}$ could be assigned to elemental Er. Angle-dispersive $\mathrm{X}$-ray diffraction (ADXRD) measurements were carried out at the powder X-ray diffraction beam line of ELETTRA synchrotron source, Trieste, Italy. For high-pressure experiments, fine-powdered ErCu was loaded in a Mao-Bell type diamond anvil cell (DAC). Hardened stainless steel gasket with a central hole of $150 \mu \mathrm{m}$ diameter and thickness $50 \mu \mathrm{m}$ contained the sample. The experimental station was based on an image plate area detector (MarResearch). The X-ray beam was collimated using an $80 \mu \mathrm{m}$ diameter pinhole. A mixture of methanol-ethanol (4:1) was used as the pressure-transmitting medium. The pressure was determined in situ by using silver powder mixed with the sample as pressure calibrant. The wavelength of the X-ray employed and the sample to image plate distance were calibrated using $\mathrm{LaB}_{6}$ diffraction pattern. X-ray diffraction patterns at various pressures were collected employing X-rays of wavelength $0.652 \AA$. The high-pressure ADXRD data were collected on the Mar345 image plate detector and read with a resolution of $100 \times 100 \mu \mathrm{m}$ pixel size up to a pressure of 23.6 GPa. The powder rings thus obtained were integrated using the versatile FIT2D software and converted to a one-dimensional $2 \theta$ vs intensity plot (Hammersley et al 1996). The lattice parameters of the sample and silver were determined by carrying out multiphase Reitveld refinement using GSAS software (Larson and Von Dreele 2004). The pressure was then determined from the equation of state of silver.

\section{Results and discussion}

The X-ray diffraction pattern of ErCu at a few representative pressures is shown in figure 1. All the diffraction peaks corresponding to the ambient cubic phase could be followed up to $23.6 \mathrm{GPa}$, the highest pressure of the present investigation. There was no appearance or disappearance of diffraction peaks, indicating the absence of structural phase transition. All the diffraction patterns were analysed using three phases (viz. sample, Ag (pressure calibrant) and SS (gasket)). Rietveld refinement was done using GSAS software (Larson and Von Dreele 2004). The typical $R_{\mathrm{p}}$ and $w R_{\mathrm{p}}$ factors of the fit were about $3 \cdot 1$ and $4.8 \%$, respectively. A representative Rietveld refinement pattern for the XRD pattern collected at $0.4 \mathrm{GPa}$ is shown in figure 2 .

The pressure variation of $V / V_{0}$ is shown in figure 3 . The second-order Birch-Murnaghan equation of state (B-M EOS) (Birch 1978) when fitted to $P-V$ data keeping pressure derivative $\left(B^{\prime}\right)$ fixed at 4.0 yielded a bulk modulus as $67.6 \mathrm{GPa}$. These values are comparable to the bulk modulus of 57.6 GPa reported for CeAg (Jun et al 2012), 66.1 GPa for YAg (Ugur et al 2009) and $70 \mathrm{GPa}$ for $\mathrm{YCu}$ (Morris et al 2004) reported by first principle calculations

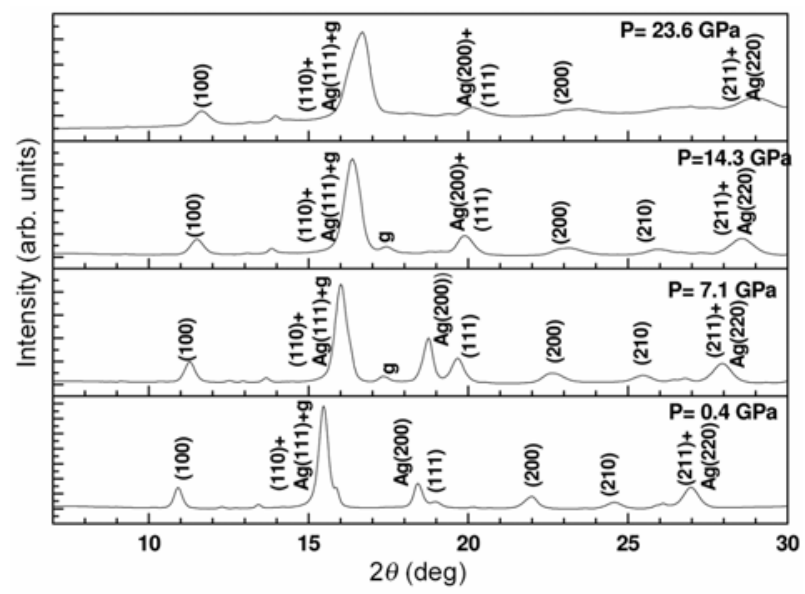

Figure 1. Pressure evolution of diffraction pattern for ErCu.

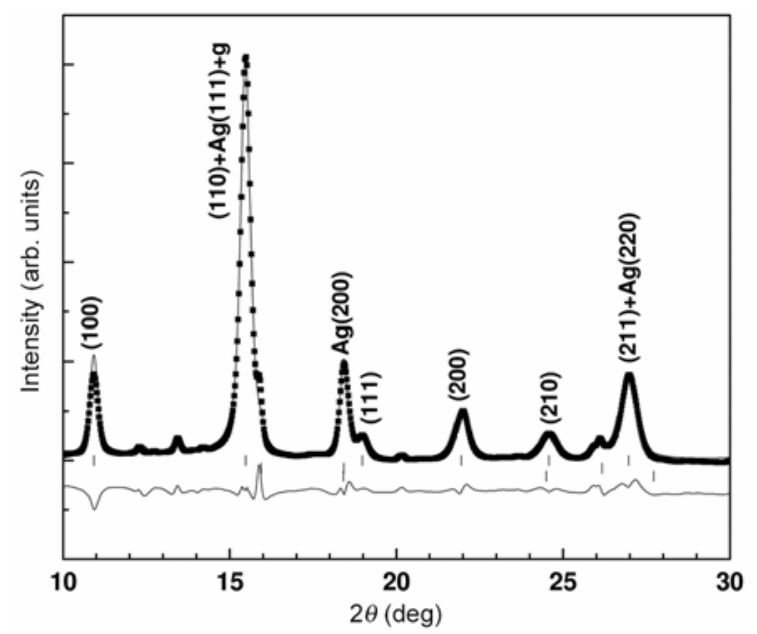

Figure 2. Rietveld refinement of angle dispersive X-ray diffraction pattern of $\mathrm{ErCu}$ at $0.4 \mathrm{GPa}$. The three descending layers of tick marks correspond to the sample, pressure calibrant (Ag) and gasket, respectively. 


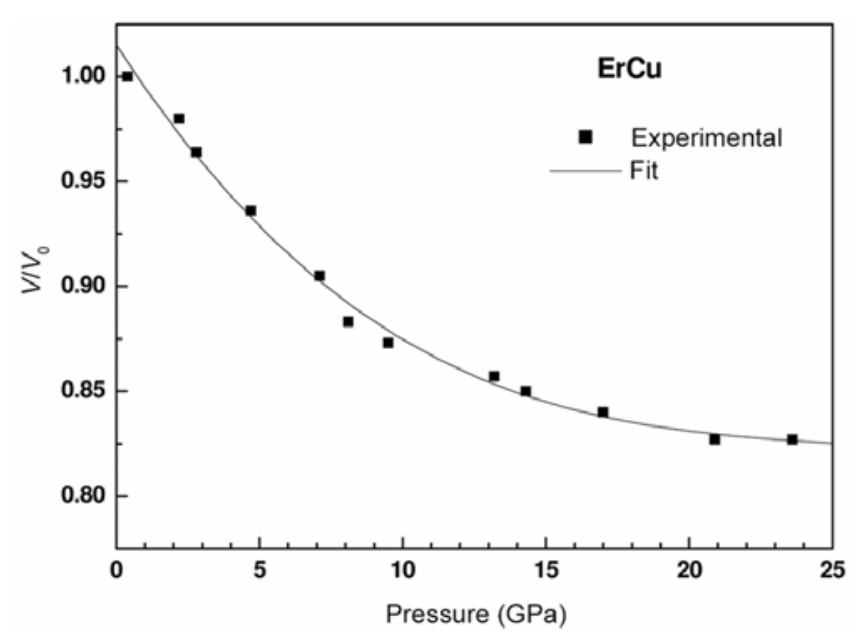

Figure 3. Equation of state for ErCu. The solid line is the second-order Birch-Murnaghan fit to the data.

and experimental bulk modulus of $76.8 \mathrm{GPa}$ for $\mathrm{HoCu}$ (Meenakshi and Garg 2012). It may be noted that the bulk modulus computed for ErCu by very recent first principles density functional calculations is $63.14 \mathrm{GPa}$, which is very close to our experimental result (Shugani et al 2013). The initial compressibility $\chi_{0}$ of $\operatorname{ErCu}\left(\chi_{0}=\right.$ $\left.1 / B_{0}=1 / 67.6 \mathrm{GPa}^{-1}\right)$ is close to the average compressibility of the $\operatorname{Er}\left(\chi_{\mathrm{Er}}=1 / 44.4 \mathrm{GPa}^{-1}\right)$ and $\mathrm{Cu}\left(\chi_{\mathrm{Cu}}=1 / 140 \mathrm{GPa}^{-1}\right)$. The results of the present investigation are also consistent with the recent study on the deformation behaviour of the analogous ductile intermetallic compound $\mathrm{DyCu}$ under compression, which shows no indications of stress-induced phase transformation or twinning (Cao et al 2011). It is interesting to note that many of the RM-type intermetallic compounds crystallizing in the rock salt structure under ambient conditions show an $\mathrm{NaCl}$ - to CsCl-type reconstructive pressure-induced structural-phase transformation, the mechanism which involves translational displacement between two adjacent layers of atoms (Chandra Shekhar and Sahu 2006; Zhou et al 2013). This indicates a better stability of the $\mathrm{CsCl}$ type structure and this has been attributed to the higher coordination number of 8 in the $\mathrm{CsCl}$ structure compared to the coordination number of 6 in the $\mathrm{NaCl}$ structure. Further structural investigations at higher pressure coupled with theoretical calculations would be helpful for further understanding of the structural stability of ErCu.

\section{Conclusions}

Our high-pressure experiments on the ductile intermetallic compound $\mathrm{ErCu}$ indicate the stability of the ambient cubic phase up to $23.6 \mathrm{GPa}$. A second-order BirchMurnaghan equation of state fit to the pressure volume data yielded a bulk modulus of $67.6 \mathrm{GPa}$ with its pressure derivative fixed at 4 .

\section{Acknowledgements}

The author would like to acknowledge the Indian DST for travel support and Italian DST for local hospitality. The help received from Dr Maurizio, Dr Alka B Garg and Dr V Vijayakumar during experiment is also acknowledged.

\section{References}

Amara M, Mourin P and Bourdarot F 1997 J. Phys.: Condens. Matter 97441

Birch F 1978 J. Geophys. Res. 831257

Blanco J A, Espeso J I, Soldevilla J G, Sal J C G, Ibarra M R, Marquina C and Fischer H E 1999 Phys. Rev. B59 512

Cao G H, Yu Z, Russell A M 2011 Mat. Sci. Eng. A528 7173

Chaboy J and Ibarra M R 1995 Phys. Rev. B52 3206

Chandra Shekar N V and Sahu P Ch 2006 J. Mater. Sci. 413207

Chouhan S S, Soni P, Pagare G, Sanyal S P and Rajagopalan M 2011 Physica B406 339

Degtyareva V F, Porsch F, Khasanov S S, Shekhtman V S and Holzapfel W B 1997 J. Alloys Compd. 46240

Demchyna R, Leoni S, Rosner H and Schwarz U 2006 Z. Kristallogr. 221420

Fleischer R L and Zabala R 1990 Met. Mat. Trans. A21 2709

Gschneidner Jr K A, Russell A, Pecharsky A, Morris J, Zhang Z, Lograsso T, Hsu D, Lo C C H, Ye Y, Slager A and Kesse D 2003 Nat. Mater. 2587

Gschneidner Jr K A 2009 Acta Mater. 5718

Hammersley A P, Svensson S O, Hanfland M, Fitch A N and Hausermann D 1996 High Press. Res. 14235

Ibarra R M, Chien T S and Pavlovic A S 1989 J. Less Common Met. 153233

Jun S Y, Lei D Y and Guang C 2012 Trans. Nonferr. Met. Soc. China 22654

Larson A C and Von Dreele R B 2004 General structure analysis system (GSAS), Los Alamos National Laboratory Report LUAR (86-748)

Meenakshi S and Garg A B 2012 J. Phys.: Conf. Series 377 2023

Morris J R, Ye Y, Lee Y B, Harmon B N, Gschneidner Jr K A and Russell A M 2004 Acta Mater. 524849

Pugh S F 1954 Philos. Mag. 45823

Shi Y, Du Y L, Chen G and Chen G L 2007 Phys. Lett. A368 495

Shugani M, Chouhan S S, Aynyas M and Sanyal S P 2013 Adv. Phys. Theor. Appl. 1983

Stoloff N S, Liu C T and Deevi S C 2000 Intermetallics 81313

Takasugi T, Masahashi N and Isumi O 1987 Acta Metall. 35381

Tao X, Chen H, Li X, Ouyang Y and Liao S 2011 Phys. Scr. 83 045301

Ugur S, Ugur G, Soyalp F and Ellialtioglu R 2009 J. Rare Earths 27664

Wang X F, Jones T E, Li W and Zhou Y C 2012 Phys. Rev. B85 134108

Wu Y, Hu W and Han S 2008 Physica B403 3792

Wu Y, Hu W and Xu L 2012 Adv. Mater. Res. 550-553 2814

Zhang Z, Russell A M, Biner S B, Gschneidner Jr K A and Lo C C H 2005 Intermetallics 13559

Zhou X, Roehl J L, Lind C and Khare S V 2013 J. Phys.: Condens. Matter 25075401 